\title{
Modification of the Brief Smell Identification Test by Introduction of a Placebo
}

\author{
Grete Kjelvik $^{1 *}$, Ole Bosnes ${ }^{2}$, Ragnhild Omli ${ }^{2}$, Liv Heidi Skotnes ${ }^{2}$, Asta K. Håberg ${ }^{3,4}$, \\ Linda R. White ${ }^{3,5}$
}

${ }^{1}$ Department of Circulation and Medical Imaging, Norwegian University of Science and Technology (NTNU), Trondheim, Norway; ${ }^{2}$ Department of Medicine (Psychiatry and Geriatrics), Namsos Hospital, Namsos, Norway; ${ }^{3}$ Department of Neuroscience, Norwegian University of Science and Technology (NTNU), Trondheim, Norway; ${ }^{4}$ Department of Medical Imaging, University Hospital, Trondheim, Norway; ${ }^{5}$ Department of Neurology, University Hospital, Trondheim, Norway.

Email: *kjelvik@ntnu.no

Received February $13^{\text {th }}, 2012$; revised March $28^{\text {th }}, 2012$; accepted April $14^{\text {th }}, 2012$

\begin{abstract}
Objective: To introduce the concept that there might be "nothing to smell" to the Brief Smell Identification Test (BSIT), with a view to masking olfactory deficits, particularly from healthy control participants in research studies. Methods: Seventy-one elderly individuals, healthy for their age, were recruited to the study. They were blindfolded and carried out a modified B-SIT where one item had been replaced with a placebo, and one odour alternative answer to three other items was replaced by the alternative "none/other" (actual odour unchanged). Results: There was no overall difference in the median or mean score achieved by the cohort compared to results obtained previously using the conventional B-SIT. The replacement of the item "turpentine" with a placebo resulted in an improved score for the item in a Norwegian setting. The overall scores were not improved. Conclusions: It is possible to introduce the concept that there may be "nothing to smell" to the B-SIT without compromising the test for healthy control individuals. This may be a more appropriate approach to olfactory testing of control individuals or patients with suspected early neurodegenerative diseases.
\end{abstract}

Keywords: B-SIT; Odor Identification; Scratch and Sniff Tests; Norwegian Population; Olfactory Testing; Neurodegenerative Diseases

\section{Introduction}

As studies of neurodegenerative conditions like Alzheimer's disease and Parkinson's disease increasingly veer towards the preclinical phase of disease, there is necessarily interest for tests that reveal early pathological changes. A change in odour identification abilities is often one of the earliest indicators of a neurodegenerative process $[1,2]$ and there is growing public awareness of this due to media coverage. The Brief Smell Identification Test (B-SIT) is a commonly-used, cross-cultural test with twelve different odours [3] that is particularly useful for testing patients with early dementia as it can usually be completed within the time window of their short-term memory.

For each odour in B-SIT, the test subject must choose an answer from four given alternative suggestions, even if they can smell nothing or do not recognise the smell. This means that subjects with little or no sense of smell that choose alternatives at random can be statistically

\footnotetext{
"Corresponding author.
}

expected to guess correctly in $25 \%$ of cases. However, one drawback of this design is that there is always "something to smell", while we have wished that the test should rather be based on the concept that there actually might be "nothing to smell". This is mainly because patients, and even healthy controls, can sometimes become angry or upset over their inability to perform a test. Where possible, and particularly in the research setting, a psychophysical test like B-SIT should ideally provide information and answers for the tester rather than for the test subject. An olfactory test where subjects have no particular expectation of odours would be preferable.

The present study was designed as a modification of B-SIT, to introduce the concept that there might be nothing to smell. This was achieved by removing an item that we have previously shown to be unsuitable in a Norwegian setting [4], and replacing it with an odourless placebo. The possibility of there being nothing to smell was also introduced to three other items as one of the alternatives. The aim of the present study was to determine whether a cohort of healthy volunteers over the age of 55 
would score as well, or perhaps better, with our modified B-SIT as we have previously found using the conventional B-SIT, and whether this might be a more appropriate approach to this type of olfactory testing for research purposes.

\section{Materials and Methods}

\subsection{Subjects}

The present study was approved by the Norwegian Data Inspectorate and the Regional Committee for Medical Research Ethics. Subjects were recruited amongst 122 elderly individuals invited to participate in the Elderly Norwegian Normative Study of the Wechsler Memory Scale-III (WMS-III) [5] and the Wechsler Adult Intelligence Scale-III (WAIS-III) (Psychological Corporation, 1997; [6], a sub-study of the third Nord-Trøndelag Health Study (HUNT3, a population study) (http://www. ntnu.edu/hunt). Due to lack of time, only 84 subjects could be invited to participate in the present study. All received oral and written information, and gave their written informed consent. Subsequently, 13 of these subjects were excluded due to neurodegenerative disease, cerebrovascular disease, Cushing's disease, chronic lung or airway disease, acute upper airway infection, nose trauma or obstruction, or acute airway allergy. Those included in the present study $(\mathrm{n}=71)$ showed no obvious deterioration of cognitive function in the preliminary interview, had no self-reported impairment in function of daily living, and were healthy relative to their age. Prior to olfactory testing, a questionnaire was used to record alcohol consumption, years and type of education, tobacco habits, use of medication, and the individual's own perception of their sense of smell. A note was made if any test individual expressed any anxiety regarding the olfactory test. Compared to an earlier study from our group [4], the present cohort was significantly younger ( 73.5 years versus 77.1 years, $p=0.002$ ) and there was a significantly higher percentage of males $(48 \%$ versus $29 \%, p=0.007)$. The demographic and WMS-III/WAISIII data are shown in Table 1.

\subsection{Olfactory Testing and Modification of B-SIT}

Olfactory testing was carried out using B-SIT (Sensonics Inc., Haddon Heights, USA) modified to introduce the possibility of there being nothing to smell. Item 2 in B-SIT, "turpentine", has been previously shown to be an odour not generally recognized in Norway [4], and this item was therefore replaced with a placebo. This consisted of a B-SIT booklet where the microcapsulated paper containing the odour for item 2 was replaced by a similar, but odourless paper. The difference was not identifiable by touch, but could be seen and for this reason
Table 1. Demographic data, cognitive test data and olfactory test (B-SIT) scores.

\begin{tabular}{ll}
\hline \multicolumn{1}{c}{ Characteristic } & \multicolumn{1}{c}{ Value } \\
\hline No. of participants & 71 \\
Gender male/female (\%) & $34(47.9) / 37(52.1)$ \\
Age: median and range (years) & $73.5,58-89$ \\
Education: median and range (years) & $12,6-20$ \\
WAIS-III FSIQ ${ }^{*}$ (mean \pm SD) & $99.9 \pm 14.7$ \\
WMS-III IM ${ }^{*}$ (mean $\left.\pm \mathrm{SD}\right)$ & $99.3 \pm 16.5$ \\
WMS-III GM ${ }^{*}$ (mean $\left.\pm \mathrm{SD}\right)$ & $105.8 \pm 16.3$ \\
B-SIT score: median and range & $10,3-12$ \\
Smoking habits (n) & \\
Non-smokers & $29(40.8 \%)$ \\
Previous smokers & $30(42.3 \%)$ \\
Occasional smokers & $2(2.8 \%)$ \\
Daily smokers & $5(7.0 \%)$ \\
Not answered & $5(7.0 \%)$ \\
Self-report on whether the experience \\
of well-known odours had changed \\
Yes
\end{tabular}

${ }^{*} \mathrm{FSIQ}=$ Full Scale IQ, IM = Immediate Memory Index, GM = General Memory Index. All index values were based on US norms of a mean $\pm \mathrm{SD}=100 \pm$ 15 .

participants were blindfolded during the test. "Turpentine" was deleted as one of the four alternative answers for item 2 and replaced with the term "none/other". This alternative answer was also introduced to three other B-SIT items, replacing the given alternative "fruit" in each case (items 1, 6 and 12, correct answers unchanged). This replacement was not expected to significantly alter the accuracy of answers as our previous study showed that "fruit" had only rarely been chosen by control individuals $(0 \%, 7.7 \%$ and $0 \%$ for items 1,6 , and 12 respectively) [4].

During the initial interview and preparation for the test, participants were warned that there might be nothing to smell and that they would be blindfolded throughout the test period.

The modified B-SIT was performed with the alternatives for each odour given orally twice; before smelling the odour, and afterwards. The written information initially given to patients made it clear that no feedback would be given during or after the test. A standardised spatula was used to release odours. 


\subsection{Statistical Analysis}

Statistical analysis was carried out using SPSS version 17.0 (SPSS Inc.; www.spss.com). Non-parametric statistical tests were applied using the Mann-Whitney U-test, Kruskal-Wallis test for multiple groups, and Kendall's rank correlation coefficient as appropriate.

\section{Results}

The median score on our modified psychophysical olfactory test (B-SIT) for healthy, elderly individuals was 10 (of a maximum of 12) (Figure 1), while the mean score was $9.8 \pm 1.8$. One individual scored only 3 , which is the minimum statistically likely if the individual cannot smell anything. One individual scored 5, two scored 6 and 2 scored 7 . All others scored 8 or above (91.5\%). There were no significant gender differences in B-SIT scores (both male and female means $10 \pm 2$ ). No test subject expressed any anxiety about using a blindfold, their olfactory ability or their performance on the modified B-SIT. The cohort recruited for this study had previously been given the WMS-III and WAIS-III tests, and several participants commented positively that by comparison the B-SIT was pleasantly short, exciting, interesting, and even fun.

Performance on each of the B-SIT items, compared to our earlier results applying the standard B-SIT, is shown in Figure 2 and Table 2. In the present study, five items (nos. 1, 5, 10, 11, 12) were correctly identified by over $90 \%$ of the participants. Changing the alternative answer "fruit" to "none/other" in items 1 and 12 therefore did not significantly alter the identification of the correct odours. The two least-correctly identified items were items 2 and 6 (respectively 53.5\% and 63.4\%), where a placebo had replaced the original odour in item 2 , and the alternative "fruit" was replaced by "none/other" in item 6 where the correct answer "rose" was unchanged. In fact, for item 2 the percentage of correct answers was actually substantially improved compared to previous results with the conventional answer "turpentine" [4], where only $21 \%$ of respondents answered the item correctly (Table 2 , $\mathrm{p}<0.0005)$, a level expected when chosen by chance. The second most frequent alternative answer given for item 2 in the present study was "soap" (23\%). Concerning item 6 ("rose"), the percentage of correct answers was reduced compared to previous data using the conventional B-SIT [4], and although not significant, showed a clear trend (Table 2, p =0.07). The second most frequent alternative answer given for item 6 in the present study was "peppermint" (27\%) and less than 3\% answered "none/other". Item 4 (correct answer "smoke") was answered correctly by over $90 \%$ in our previous study [4], but by only $74.6 \%$ in the present study, despite the item being unchanged. This difference was significant (Table 2, $\mathrm{p}=0.028$ ).

The total B-SIT scores of smokers and previous smokers compared to non-smokers, were not significantly different $(\mathrm{p}>0.1$, Kruskal-Wallis Test). When asked whether their experience of well-known odours had changed, the two individuals scoring lowest with the

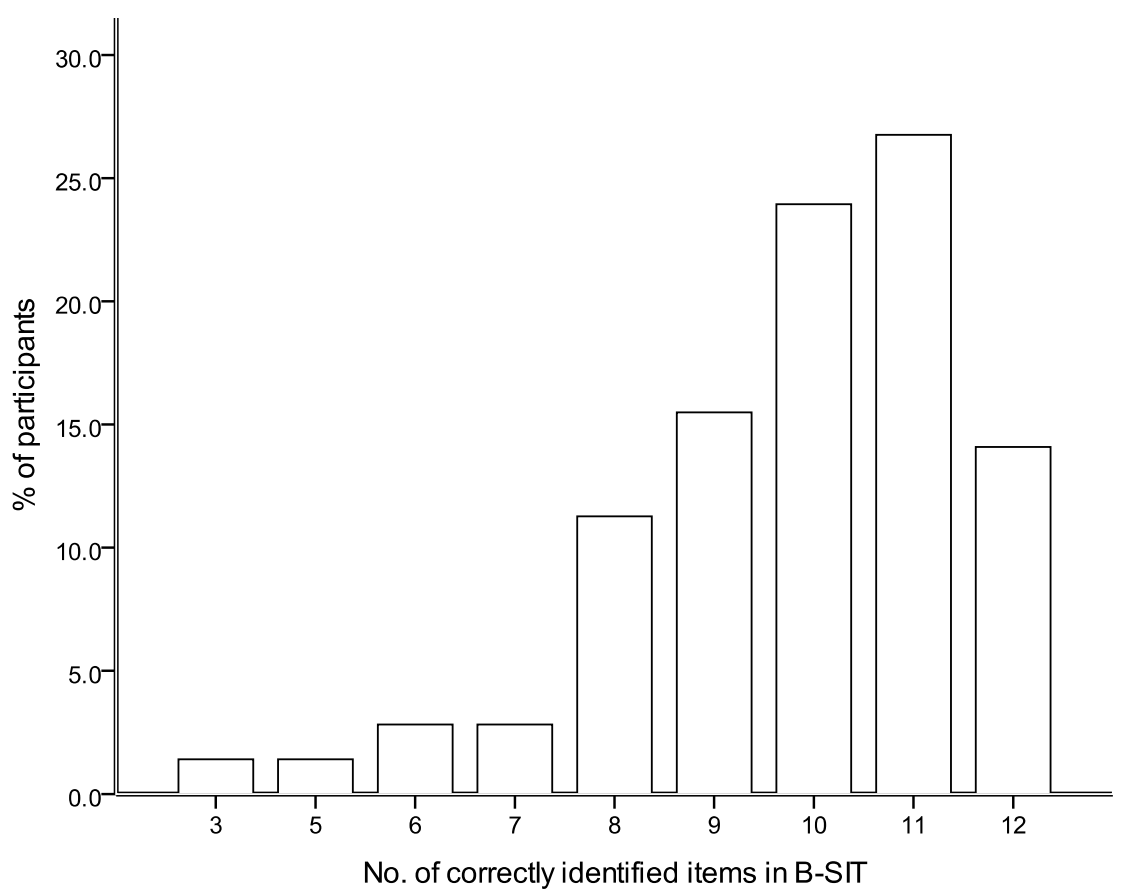

Figure 1. The histogram shows the range of scores of the modified psychophysical olfactory test (B-SIT) for healthy elderly individuals. 


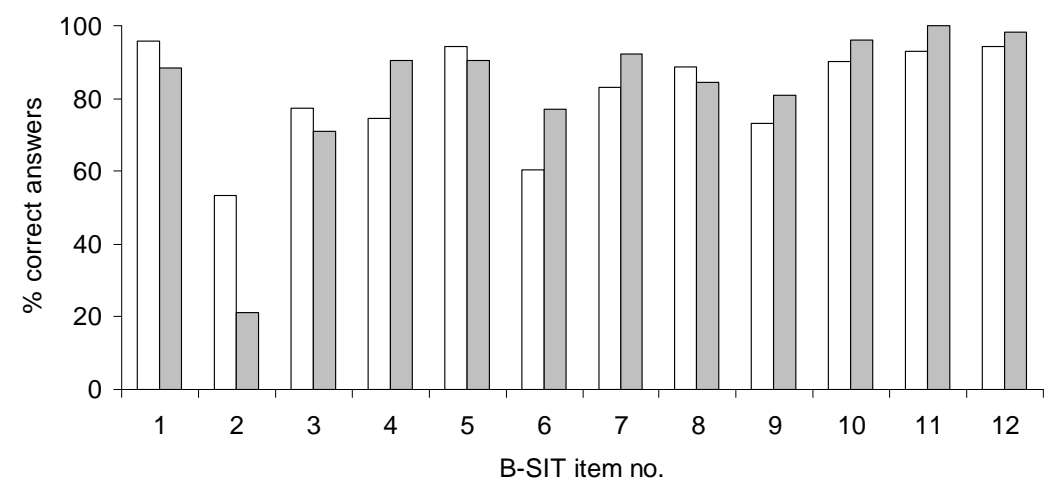

Figure 2. Performance on each of the B-SIT-items is shown as the percent of correct answers for the modified version of the test (white), and for results obtained previously using the standard B-SIT by Kjelvik et al. 2007 (grey).

Table 2. The diagnostic accuracy of the modified version of the Brief Smell Identification Test (B-SIT) is shown as Group 1, whereas Group 2 represents data obtained previously with the standardized version of the test. The percentage of correct answers for each group is shown for all twelve B-SIT items.

\begin{tabular}{cccc}
\hline $\begin{array}{c}\text { B-SIT } \\
\text { item no. }\end{array}$ & Odorant & $\begin{array}{c}\text { \% correct answers } \\
\text { Group 1 }\end{array}$ & $\begin{array}{c}\text { \% correct } \\
\text { answers Group 2 }\end{array}$ \\
\hline 1 & Cinnamon & 95.8 & 88.5 \\
2 & Turpentine/no odour* & $53.5^{* * *}$ & 21.2 \\
3 & Lemon & 77.5 & 71.2 \\
4 & Smoke & $74.6^{* * *}$ & 90.4 \\
5 & Chocolate & 94.4 & 90.4 \\
6 & Rose & 63.4 & 76.9 \\
7 & Paint thinner & 83.1 & 92.3 \\
8 & Banana & 88.7 & 84.6 \\
9 & Pineapple & 73.2 & 80.8 \\
10 & Gasoline & 90.1 & 96.2 \\
11 & Soap & 93.0 & 100 \\
12 & Onion & 94.4 & 98.1 \\
\hline
\end{tabular}

*Kjelvik et al. 2007; ${ }^{* *}$ Odour no. 2 turpentine was replaced with a placebo in the modified version of the B-SIT; ${ }^{* * *}$ Significant difference between groups, $\mathrm{p}<0.05$ (Mann-Whitney U test).

modified B-SIT ( 3 and 5 correct answers) both answered "no", as did one each of the individuals scoring 6 and 7.

A clear negative correlation was found between age and the modified B-SIT as a whole $(\mathrm{r}=-0.36, \mathrm{p}<$ 0.0005 ). A weak (though not significant) negative association was found between age and number of years of education $(r=-0.17, p=0.064)$, but no trend was seen between education and the modified B-SIT score. Additionally, positive correlations were found between the modified B-SIT score and all sections of WAIS-III and WMS-III (WAIS-III FSIQ: $r=0.21, p=0.017$, WMS-III
IM: $r=0.26, p=0.004$, WMS-III-GM: $r=0.23, p=$ $0.01)$.

\section{Discussion}

The current results suggest that modifying B-SIT to include the concept that there is nothing to smell by introducing a placebo as one of the alternatives, does not change the mean or median score achieved by a cognitively healthy, elderly test cohort, compared to previous studies $[4,7,8]$. That the results with the modified B-SIT correlated significantly with all sections of WAIS-III and WMS-III supports the quality of the overall data, and that the modifications made to the B-SIT did not reduce its applicability as a rapid olfactory test. Over $90 \%$ of participants scored 8 or over in the modified B-SIT, which was also found to be the best cut-off to distinguish between healthy controls and patients with Alzheimer's disease in an earlier study [4]. In a Norwegian setting, this can be achieved quite easily by replacing the poorlyrecognised item 2, "turpentine" with a placebo. The replacement of the answer alternative "fruit" by "none/ other" in three other items (1,6 and 12) without altering the correct answers, also permits repetition of the concept that there may be nothing to smell. Although there were fewer correct answers overall for item 6 in the present study, this trend seemed to be related more to the participants' assessment of the odour emitted by the test, rather than the modification to the alternative answers. This is further supported by the lower number of correct answers to item 8 than found previously [4], even though this item was unchanged. One factor that may have influenced the reduction in correct answers to these items is the fact that there were a higher percentage of males in the percent study compared to our previous study [4]. It has been established that females perform fractionally better on B-SIT than males [9].

Although the replacement of item 2 with a placebo significantly improved the number of correct answers for the item, it was still the item with the lowest number of 
correct answers. The most popular wrong answer was "soap", which may reflect the faint odour that is found in the paper of new B-SIT booklets. It seems unlikely that it will be easy to significantly improve on the overall score on B-SIT with a healthy elderly test group.

Commercial olfactory tests are increasingly used as additional neuropsychological support for the diagnosis of neurodegenerative diseases like Alzheimer's or Parkinson's disease [10]. However, deterioration of odour identification is not a specific indication of neurodegeneration, and can be a consequence of increasing age or nasal trauma $[9,11]$, or the result of an environmental factor like smoking $[12,13]$. In the present study with a cohort of 71 individuals, no significant differences were found between the results from the B-SIT and smoking habits, probably because the cohort was not large enough to find differences between small subgroups. Few individuals in this study demonstrated a poor odour identification ability, but it is clear that not all were aware of their problem. However, none of those with a smell deficit reacted negatively to the modified B-SIT.

A strong correlation was found between B-SIT and age, which strengthens the overall validity of the results. A trend towards a negative correlation between age and education is in accordance with improving standards of education in Norway during the last 60 years.

In conclusion, it is possible to introduce the concept of there being "nothing to smell" to the B-SIT by simple means, and the results with a healthy, elderly cohort suggest that the values obtained (at least in Norway) are not significantly reduced compared to use of the conventional B-SIT. We feel this is a more acceptable approach to olfactory testing in research studies where psychophysical tests like B-SIT are often applied to healthy control individuals and patients with suspected early neurodegenerative diseases.

\section{REFERENCES}

[1] C. Hawkes, "Olfaction in Neurodegenerative Disorder," Advances in Oto-Rhino-Laryngology, Vol. 63, 2006, pp. 133-151. doi:10.1159/000093759

[2] T. Kovacs, "Mechanisms of Olfactory Dysfunction in Aging and Neurodegenerative Disorders," Ageing Re- search Reviews, Vol. 3, No. 2, 2004, pp. 215-232. doi:10.1016/j.arr.2003.10.003

[3] R. L. Doty, "The Brief Smell Identification Test Admninstrator Manual," Sensonics Inc., Haddon Heights, 2001.

[4] G. Kjelvik, S. B. Sando, J. Aasly, K. A. Engedal and L. R. White, "Use of the Brief Smell Identification Test for Olfactory Deficit in a Norwegian Population with Alzheimer's Disease," International Journal of Geriatric Psychiatry, Vol. 22, No. 10, 2007, pp. 1020-1024. doi: $10.1002 /$ gps. 1783

[5] D. Wechsler, "Wechsler Memory Scale, Third Edition, WMS-III," The Psychological Cooperation, San Antonio, 1997.

[6] D. Wechsler, "Wechsler Adult Intelligence Scale, Third Edition, WMS-III," The Psychological Cooperation, San Antonio, 1997.

[7] R. S. Wilson, S. E. Arnold, J. A. Schneider, P. A. Boyle, A. S. Buchman and D. A. Bennett, "Olfactory Impairment in Presymptomatic Alzheimer's Disease," Annals of the New York Academy of Sciences, Vol. 1170, 2009, pp. 730-735. doi:10.1111/j.1749-6632.2009.04013.x

[8] G. E. Swan and D. Carmelli, "Impaired Olfaction Predicts Cognitive Decline in Nondemented Older Adults," Neuroepidemiology, Vol. 21, No. 2, 2002, pp. 58-67. doi:10.1159/000048618

[9] R. L. Doty, P. Shaman, S. L. Applebaum, R. Giberson, L. Siksorski and L. Rosenberg, "Smell Identification Ability: Changes with Age," Science, Vol. 226, No. 4681, 1984, pp. 1441-1443. doi:10.1126/science. 6505700

[10] M. Wolfenberger and I. Schneiper, "Sniffin Sticks: A New System for Olfactory Assessement in Routine Clinical Practice," HNO, Vol. 47, No. 7, 1999, pp. 629-636. doi: $10.1007 / \mathrm{s} 001060050436$

[11] K. A. Seiberling and D. B. Conley, "Aging and Olfactory and Taste Function," Otolaryngologic Clinics of North America, Vol. 37, No. 6, 2004, pp. 1209-1228. doi:10.1016/j.otc.2004.06.006

[12] M. Katotomichelakis, D. Balatsouras, G. Tripsianis, S. Davris, N. Maroudias, V. Danielides and C. Simopoulos, "The Effect of Smoking on the Olfactory Function," Rhinology, Vol. 45, No. 4, 2007, pp. 273-280.

[13] R. E. Frye, B. S. Schwartz and R. L. Doty, "Dose-Related Effects of Cigarette Smoking on Olfactory Function," Journal of the American Medical Association, Vol. 263, No. 9, 1990, pp. 1233-1236. doi:10.1001/jama.1990.03440090067028 